\title{
MULTI-AGENT SIMULATION OF ATTACKS AND DEFENSE MECHANISMS IN COMPUTER NETWORKS
}

\author{
Igor Kotenko \\ St.-Petersburg Institute for Informatics and Automation of Russian Academy of Sciences \\ 39, $14^{\text {th }}$ Liniya, St. Petersburg, 199178, Russia \\ ivkote@iias.spb.su, http://www.comsec.spb.ru/kotenko/
}

\begin{abstract}
The paper considers the approach to investigation of distributed cooperative cyber-defense mechanisms against network infrastructure oriented attacks (Distributed Denial of Service, network worms, botnets, etc.). The approach is based on the agent-based simulation of cyber-attacks and cyber-protection mechanisms which combines discrete-event simulation, multi-agent approach and packet-level simulation of network protocols. The various methods of counteraction against cyber-attacks are explored by representing attack and defense components as agent teams using the software simulation environment under development. The teams of defense agents are able to cooperate as the defense system components of different organizations and Internet service providers (ISPs). The paper represents the common framework and implementation peculiarities of the simulation environment as well as the experiments aimed on the investigation of distributed network attacks and defense mechanisms.
\end{abstract}

Keywords: cyber-attacks, cyber-defense, agents, simulation.

\section{INTRODUCTION}

Today we are in growing dependence from information and telecommunication technologies. The further use of them becomes impossible without appropriate protection mechanisms and effective homeland security solutions. The important problem in homeland security which solution is urgently needed is the investigation of counteraction between malefactors and defense systems in computer networks, including the Internet, and the creation of prospective intelligent cyber-defense systems [20].

The design and implementation of effective cyber-defense intelligent cyber-defense system is a very complicated problem. According to our view the prospective network cyber-defense systems has to be fully integrated and multi-echeloned ones. To effectively detect the computer attacks or unauthorized operations and to flexibly react on them, it is needed to carry out the continuous control of network functioning, analyze possible risks, collect knowledge about counteraction, detection and reaction methods and use them for defense reinforcement. Besides, the effective cyber-defense should include the mechanisms of attack prevention, detection, source tracing and protection as well as can only be achieved by the cooperation of different distributed components [17, 18]. For example, detection of Distributed Denial of Service (DDoS) flooding attack $[3,12,16,23,24,27]$ is most accurate close to the victim, but separation of legitimate is most successful close to the sources, therefore the security sub-systems (or teams) have to be located at different network places and tightly cooperate (Fig. 1).



Fig. 1 - Cooperation of agent teams in the Internet.

The cyber-defense systems have to be adaptive and evolve dynamically with the change of network conditions. To implement these possibilities in prospective cyber-defense system one must implement the dynamic behavior, autonomy and adaptation of particular components, the use of 
methods based on negotiations and cooperation that lie in the basis of multi-agent systems and (or) autonomic computing.

Furthermore, the prospective cyber-defense system has to provide at least three levels of cybersecurity (Fig. 2).

\begin{tabular}{|c|c|c|c|c|}
\hline \multicolumn{5}{|c|}{ Intelligent cyber-defense management } \\
\hline $\begin{array}{c}\text { Evaluation } \\
\text { of network state }\end{array}$ & \multicolumn{2}{|c|}{$\begin{array}{c}\text { Generation of defense } \\
\text { mechanisms }\end{array}$} & & Adaptation \\
\hline \multicolumn{5}{|c|}{ Proactive cyber-defense mechanisms } \\
\hline Security assessme & \multicolumn{2}{|c|}{$\begin{array}{l}\text { Attack detection and } \\
\text { counteraction }\end{array}$} & & $\begin{array}{l}\text { factor deception, } \\
\text { etc. }\end{array}$ \\
\hline \multicolumn{5}{|c|}{ “Traditional” cyber-defense mechanisms } \\
\hline $\begin{array}{l}\text { Cryptographic } \\
\text { protection }\end{array}$ & Access control & \multicolumn{2}{|c|}{ Integrity control } & Auditing, etc. \\
\hline
\end{tabular}

Fig. 2 - Levels of cyber-security.

First level contains "traditional" static cyberdefense mechanisms implementing identification and authentication, cryptographic protection, access control, auditing, network filtering, etc.

Second level includes proactive cyber-defense mechanisms that provide information collection, security assessment, network state monitoring, attack detection and counteraction, malefactor deception, etc.

Third level corresponds to cyber-defense management that fulfills the integral evaluation of network state, the choice of adequate or optimal defense mechanisms and their adaptation. This level is built on top of various non-adaptive security mechanisms, which makes it applicable for a wide range of cyber defenses.

The paper considers the approach to multi-agent simulation of cyber-attacks and distributed cooperative multi-level cyber-defense for the exploration of prospective intelligent cyber-defense systems. We analyze various methods of counteraction against cyber-attacks by representing attack and defense components as agent teams using the simulation environment developed. Various teams of defense agents are able to cooperate as the defense system components of different organizations and Internet service providers (ISPs).

The rest of the paper is structured as follows. Section 2 outlines the common multi-agent modeling and simulation framework suggested. Section 3 describes the implementation peculiarities of the simulation environment developed. Section 3 presents demonstrates the examples of experiments provided with the simulation environment. Conclusion surveys the main results of the paper.

\section{SIMULATION FRAMEWORK}

The multi-agent approach to simulation supposes that the cyber-counteraction is represented as the interaction of different teams of software agents [17, 18]. The aggregated system behavior becomes apparent by means of local interactions of particular agents in dynamic environment that is defined by the model of computer network.

There are at least three different classes of agent teams [19]:

- teams of agents-malefactors,

- teams of defense agents,

- teams of agents-users.

Agents of different teams can be in indifference ratio, cooperate or compete up till explicit counteraction. Agents are supposed to collect information from various sources, operate incomplete knowledge, forecast the intentions and actions of other agents, try to deceive the agents of competing teams, react to actions of other agents. Every team member might have different information about actions done by other team members.

Therefore, the model of agent behavior must be able to represent the incompleteness of information and the possibility of accidental factors. Besides, the agent behavior depends on information that the team has and on its distribution on the set of particular agents. The models of agent functioning are to foresee, what each agent knows, what task has to be solved and to which agent it must address its request to receive such information if it is outside of its competence.

The general conceptual model of cybernetic agents' counteraction and cooperation includes (Fig. 3) [8, 19]:

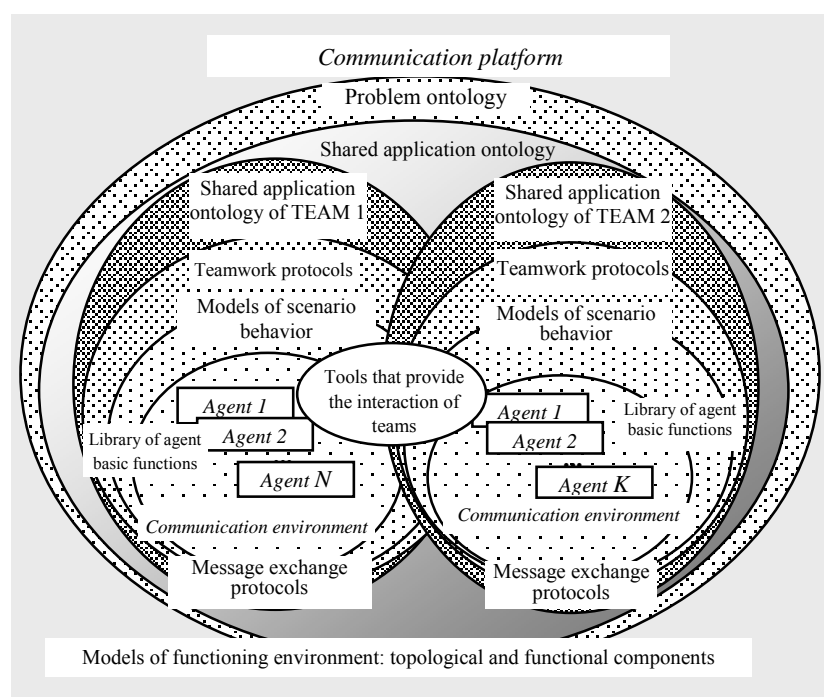

Fig. 3 - Abstract model of team interaction.

- Ontology of application domain containing application notions and relations between them (we differentiate the problem ontology, 
the shared application ontology, the application ontology of particular team and particular agent);

- Protocols of teamwork for the agents of different teams;

- Models of scenario behavior of agents for team, group and individual levels;

- Libraries of agent basic functions;

- Communication platform and components for agent message exchange;

- Models of functioning environment, including topological, functional and other components;

- Models that provide the interaction of teams (antagonistic and non-antagonistic competing or various kinds of cooperation).

As for every application domain the information security ontology represents the partially normalized set of notions that are to be used by other agents. The given ontology defines the subset of notions that various agents use for cooperative solving of stated tasks. Each agent uses a certain part of application domain ontology. Each agent specialization is represented by a subset of ontology nodes.

Attack agents are subdivided at least into two classes:

- "Demons" and

- "Masters".

To simulate distributed cooperative defense, the security agents belong to the following classes:

- Information processing ("samplers");

- Attack detection ("detectors");

- Filtering and balancing ("filters");

- Traffic limiting ("limiters");

- Traceback and investigation ("investigators").

It is supposed that agents are to be able to realize the mechanisms of self-adaptation and evolution. The team of agents-malefactors evolves with the aid of generating new attacks and attack scenarios to overcome the defense. The team of defense agents adapts by changing the security policy and defense methods and profiles.

The main basis for the research is the agent teamwork approaches: joint intentions theory [4], shared plans theory [9] and the hybrid approaches [31].

Another fundamental component of the research is represented by the studies on reasoning systems about opponent intentions and plans [2, 7, 32, 34]. The important components in this research are the methods of reflexive processes theory [21], game theory and control in conflict situations [5].

The teams of malefactors and defense agents are to adapt to network reconfiguration, traffic changes and new types of defense and attacks on the basis of past experience. Therefore it is important to take into account the present studies in the area of adaptation
[30], agent learning $[1,6,10,35]$, autonomic computing $[11,15,33]$, and combining artificial immune systems with different computational intelligence methods, such as fuzzy systems, neural networks, etc. $[13,25]$.

\section{SIMULATION ENVIRONMENT}

A multi-level software environment was supposed to be developed to implement the proposed approach. It differs from the known tools for agentoriented simulation, as the basis for simulation the tools should be used which provide adequate simulation of network processes.

The spectrum of possible approaches to modeling and simulation are differentiated from analytical to scaled-down and full-scale (see Fig. 4) [29].

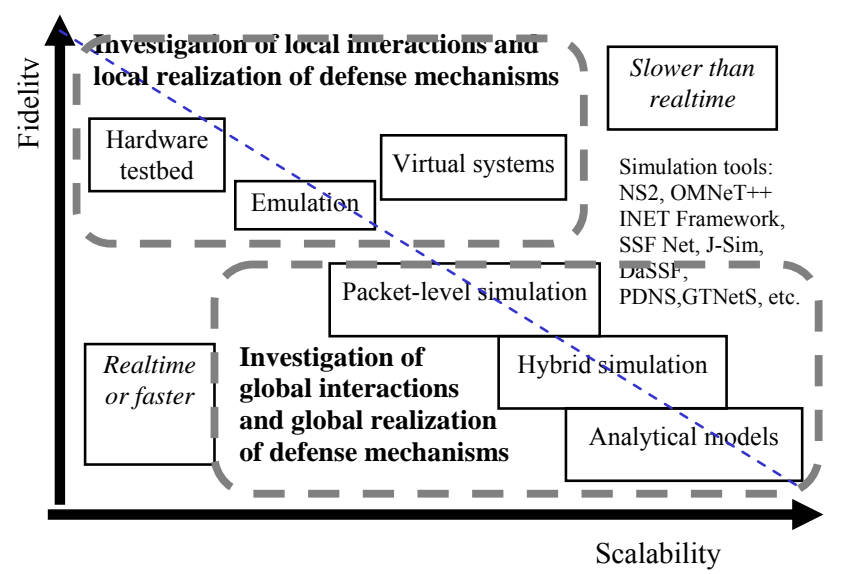

Fig. 4 - Variety of used models.

The choice of model depends on the needed fidelity and scalability of simulation.

The scalability is defined as number of network host (client hosts and routers) which can be simulated using the given method.

The fidelity is defined as a degree of network and hosts destabilization used.

Analytical models allow to simulate large-scale Internet processes (including DDoS attacks and worms epidemics) but these models describe the processes only on abstract level.

Packet-level simulation allows enough adequate rendering of such processes. The defense and attack actions are represented as the exchange of packets. This allows the high-fidelity simulation of datalink, network, transport and application layers.

The highest fidelity is reached on the hardware testbeds, but the size of simulated network is restricted enough.

We have chosen the packet-based approach as it provides acceptable scalability and fidelity. In Fig. 4, various program simulators which can be 
used for simulation are depicted: NS2, OMNeT++ INET Framework, SSF Net, J-Sim, etc.

To choose the necessary tool we fulfilled the detailed analysis of these simulation environments [18] and OMNeT++ INET Framework was chosen [26].

The simulation environment architecture suggested includes the following components (Fig. 5):

- Simulation Framework,

- Internet Simulation Framework,

- Multi-agent Simulation Framework,

- Subject Domain Library.

Simulation framework is a discrete event simulator. Other components are expansions or models for Simulation Framework.

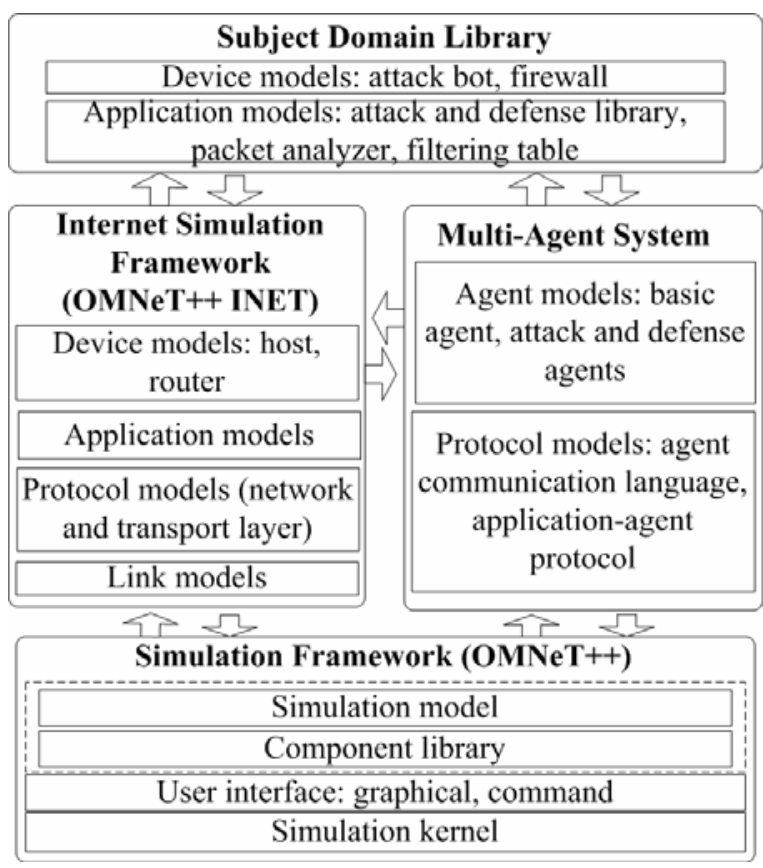

Fig. 5 - Simulation environment architecture.

Internet Simulation Framework is a modular simulation suite with a realistic simulation of Internet nodes and protocols. The highest IP simulation abstraction level is the network itself, consisting of IP nodes. IP node corresponds to the computer representation of Internet Protocol. IP node can represent router or host. IP node in Internet Simulation Framework corresponds to the computer representation of Internet Protocol. The modules of IP node are organized as operating system process IP datagram. The module that is responsible for the network layer (implementing IP processing) and the "network interface" modules are mandatory. In addition one can plug the modules that implement higher layer protocols.

Multi-agent Simulation Framework allows realizing agent-based simulation. It consists of modules representing the intelligent agents implemented as applications. There were used the elements of abstract FIPA architecture during agent modules design and implementation. Agent communication language is implemented for the agent interactions. The message transmission occurs above the TCP protocol (transport layer) implemented in Internet Simulation Framework. Agent directory is mandatory only for agent that coordinates other agents in its team. Agent can control the other modules due to messages.

Subject Domain Library is the library used for imitation of processes from subject domain and containing modules that extend functionality of IPhost: filtering table and packet analyzer.

This architecture was implemented for multiagent simulation DDoS attack and defense mechanisms with the use of OMNeT++ INET Framework and software models developed in $\mathrm{C}++$.

Agent models implemented in Multi-agent Simulation Framework are represented with generic agent, attack and defense agents.

Subject Domain Library contains various models of hosts, e.g. attacking host, firewall etc., and also the application models (attack and defense mechanisms, packet analyzer, filtering table).

Fig. 6 shows the multi-window user interface of the simulation environment.

The management window has the time axis with the system events: opening or closing the TCP connection, attack signals, defense acts, etc.

The simulated network window depicts the network hosts and channels. Hosts can fulfill different functionality depending on their parameters or a set of internal modules. Internal modules are responsible for functioning of protocols and applications at various levels of OSI model. Hosts are connected by channels which parameters can be changed. Applications (including agents) are established on hosts. Applications are connected to corresponding modules of protocols.

The structure of generic host is depicted on the bottom left. The deployed agent is represented as the blue symbol of human in the frame.

The environment allows to examine the different information describing the simulation functioning. For example, the diagram that shows the change in network parameters is depicted at the top right.

The networks used for simulation consist of various subnets that are, for instance, the regions of responsibility of various ISPs.

For example, one can mark out the defense subnet where the attack victim is located, the intermediate subnets where the standard hosts generate generic network traffic, and attack subnets where the attack agents are located.

The networks are built with the methods of 


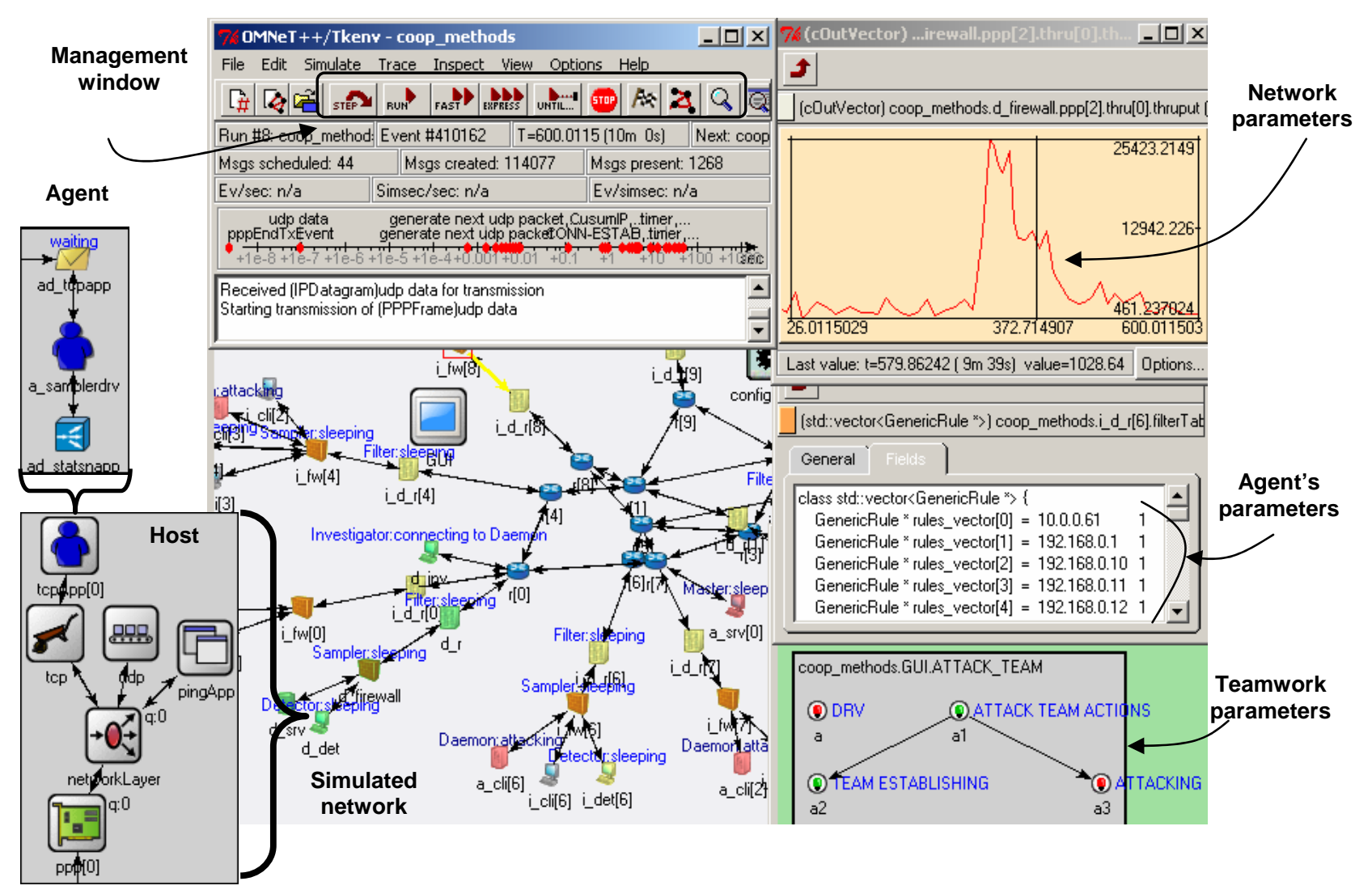

Fig. 6 - Multi-window user interface of environment.

generating topologies that are close to the real Internet [22].

\section{EXPERIMENTS}

The developed simulation environment allows carrying various experiments aimed to investigate attacks and prospective defense strategies. One can vary network topology and configuration, structure and configuration of attack and defense teams, attack and defense mechanisms, team cooperation parameters etc. The evaluations of various effectiveness parameters of defense mechanisms are done on the basis of experiments results. The analysis of applying conditions is also fulfilled for these parameters.

The attack parameters used in the experiments are as follows: Victim type - host (server that provides some service); Attack type - brute-force; Impact on the victim - disruptive; Attack rate dynamics - constant, variable; Agents' set permanency - constant, variable; Possibility of exposure - discoverable filterable attack; Source addresses validity - valid (real), spoofed: random, subnet; Degree of automation - semi-automatic with direct communication.

In the experiments we have used three defense methods: Hop counts Filtering (HCF) [14], Source IP address monitoring (SIPM) [28] и Bit Per Second (BPS). HCF consists in building the tables of subnets and amount of hops till them in the learning mode. Attack is found out on the basis of amount of hops differing from received in learning mode. SIPM uses the assumption that during attack a lot of new IP addresses appear. BPS allows detecting the attacker due to exceeding the normal traffic threshold.

The other defense parameters are as follows: Deployment location - intermediate, defended subnets; Covered defense stages - attack prevention, attack detection, attack source detection, attack counteraction; Attack source detection technique can detect when source address is not spoofed; Attack prevention technique - packet filtering; Technique for gathering of model data - learning; Determination of deviation from model data: thresholds (HCF, BPS), determination of fluctuation in probabilistic traffic parameter (SIPM).

Let us consider two examples of experiments fulfilled where we analyzed different modes of DDoS attacks and defense:

- Experiment 1: Investigation of simple adaptation of attack and defense teams.

- Experiment 2: Investigation of different cooperation modes between defense teams.

Let us consider the values of the main parameters that define the computer network models, attack and defense mechanisms that are used for the experiments.

To create a topology for testing, we used the generator of networks that are close to the real 
Internet networks. The following basic network topology parameters were set: minimum amount of connection is 2 , the amount of routes in simulated networks is 10 , the probabilistic value $\gamma=2.25$ [22]. Routers are connected with the fiber-glass data channels, propagation delay is 1 microsec; datarate is $2488 \mathrm{Mbit}$. Other hosts are connected by Ethernet data channels, propagation delay is 0.1 microsec; datarate is 100 Mbit.

Clients are randomly connected to the routers of the basic network. The amount of clients is an input parameter for experiments (its initial value is 10). The defended server is d_srv. The basic parameters of network clients are as follows: server address "d srv"; server port - 80; start time is a random value with the exponential probability distribution function (PDF) and mean $5 \mathrm{sec}$; one request per session is used; request length is a random value with normal PDF with mean 350 and dispersion 20 bits; reply length is a random value with exponential PDF and mean 2000 bits; Think time is a random value with normal PDF with mean 2 and dispersion $3 \mathrm{sec}$; Idle interval is a random value with normal PDF with mean 36 and dispersion $12 \mathrm{sec}$; Reconnect interval is $30 \mathrm{sec}$.

The fragment of the network which was used in the first experiment is depicted in Fig. 7.

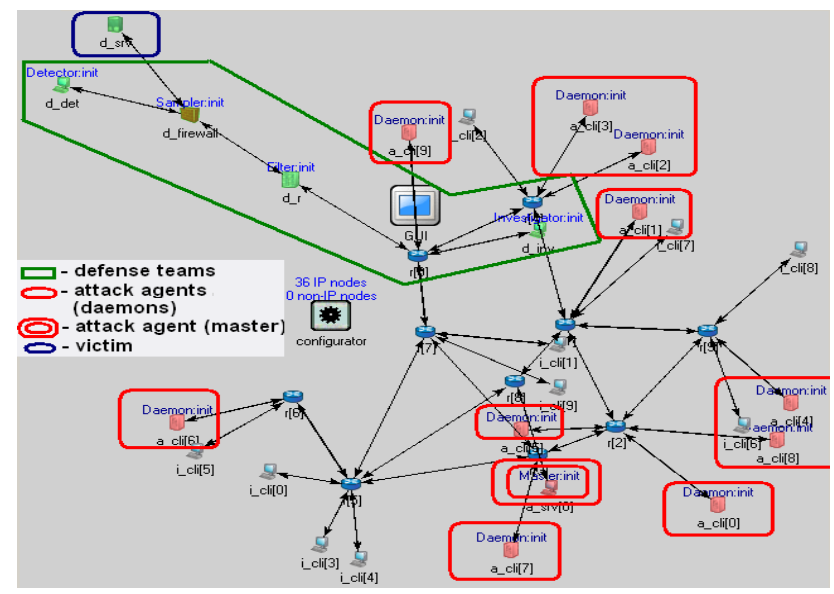

Fig. 7 - Experiment 1: the Internet fragment and agent teams.

- The fragment of decision making and acting sequence is as follows (Fig 8):

- Normal work of users (interval $0-300$ seconds).

- Defense team: formation of the team; the team start using BPS method.

- Attack team: formation of the team; after 300 seconds the team begins the attack actions (intensity of attack for every daemon - 0.5 , no IP spoofing).

- Defense team: data processing, attack detecting (using BPS) and reacting (interval $300-350$ seconds); blocking the attack, destroying some attack agents (interval $300-600$ seconds).

- Attack team: after 600 seconds the automatic adaptation is fulfilled (redistributing the intensity of attack (0.83), changing the method of IP spoofing (Random)).

- Defense team: data processing, failing to detect the attack (using BPS method) - Detector sees that the input channel throughput has noticeably lowered, but does not receive any anomaly report from sampler because BPS does not work.

- Defense team: Changing defense method on SIPM (automatic adaptation); Data processing, attack detecting (using SIPM method) and reacting - (interval $600-700$ seconds).

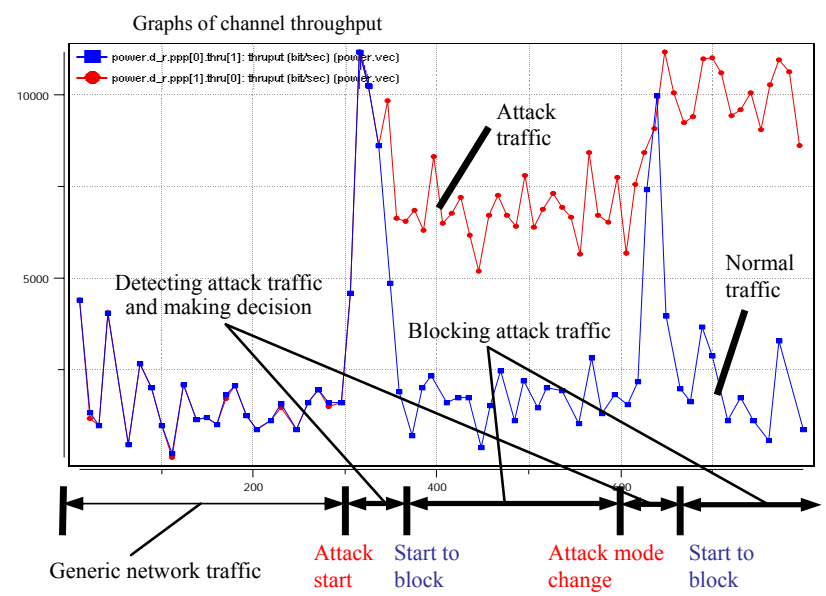

Fig. 8 - Scheme of teams' acting.

The fragment of the network which was used in the second experiment is depicted in Fig. 9.

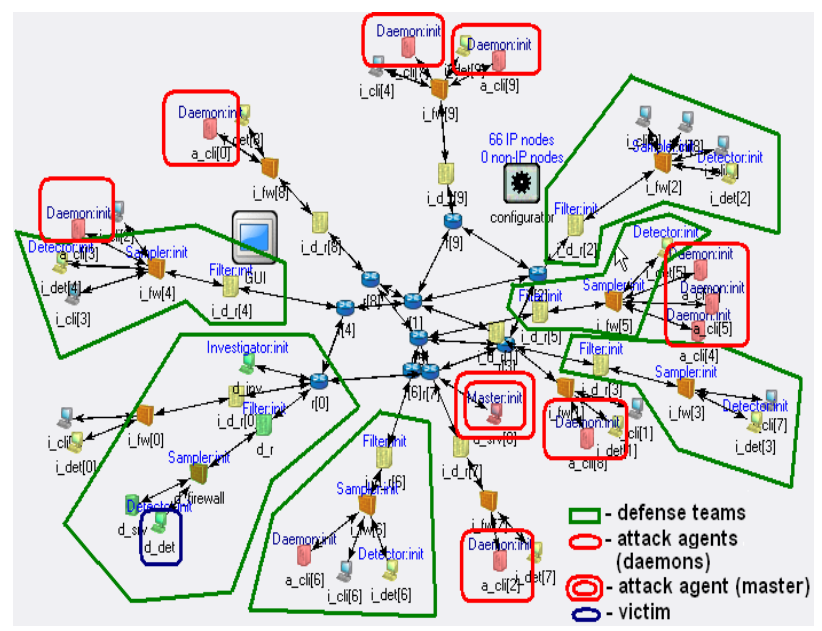

Fig. 9 - Experiment 2: the Internet fragment and agent teams.

We investigated the models of cooperation between distributed defense teams:

(1) filter-level cooperation: the team whose network is under attack can apply filtering rules on 
the filters of other teams;

(2) sampler-level cooperation: the team whose network is under attack can get the traffic information from the samplers of other teams;

(3) "poor" cooperation: the teams can get the traffic information from the samplers of some other teams and apply filtering rules on the filters of some other teams (each team knows a subset of other teams depending on the cooperation degree);

(4) "full" cooperation: the team whose network is under attack can get the traffic information from all samplers of other teams and apply filtering rules on all filters of other teams.

Fig. 10 depicts the volume of input traffic before and after the filter of the team which network is under attack when the BPS method is used.
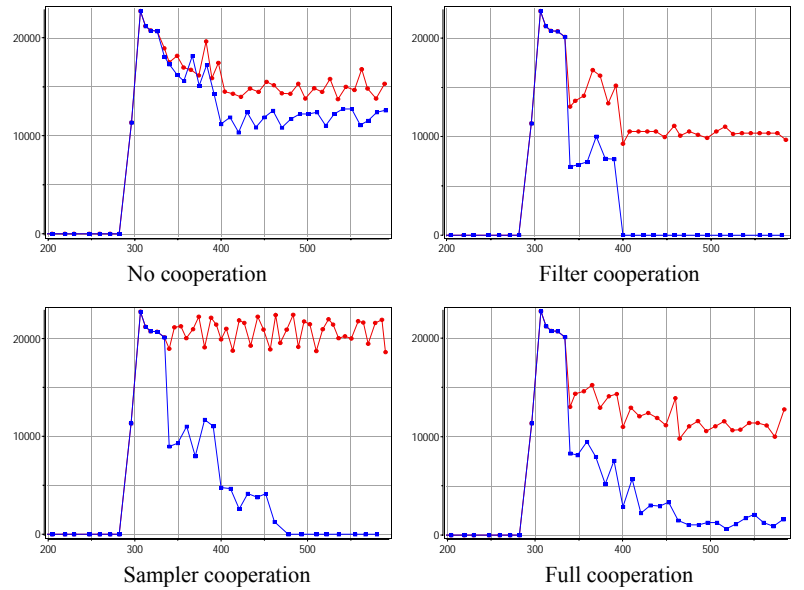

Fig. 10 - Volume of input traffic before and after the filter.

The other effectiveness and efficiency parameters of different defense mechanisms which were investigated are as follows: rate of dropped legitimate traffic (false positive rate); rate of admitted attack traffic (false positive rate); attack reaction time.

These parameters were investigated in dependence on the following input parameters: network configuration; attack intensity; IP address spoofing technique used in attack; internal parameters of defense mechanisms and their combinations; quantity and distribution of defense teams, etc.

The best cooperative schema on the basis of output parameters is "full cooperation". Samplersagents cooperation played the crucial role in defense. It causes the permanent traffic data exchange between various defense teams.

\section{CONCLUSION}

This paper considered the approach to investigation of distributed cooperative cyber- defense mechanisms against network attacks. The approach is based on the simulation of cyber-attacks and cyber-protection mechanisms which combines discrete-event simulation, multi-agent approach and packet-level simulation of network protocols.

A lot of different experiments were carried. They were aimed to investigate dependence of defense mechanisms effectiveness parameters from network topology and configuration, structure and configuration of attack and defense teams, attack and defense mechanisms and defense teams' cooperation. Experiments showed that team cooperation leads to the essential defense effectiveness improvement.

Future work is related with more thorough investigation of effectiveness of cooperation mechanisms for different teams and inter-team interaction of agents, implementation of selfadaptation and self-learning of agents. We are planning to expand the attacks and defenses library, elaborate particular components functionalities.

\section{ACKNOWLEDGMENT}

This research is being supported by grant of Russian Foundation of Basic Research (Project No. 07-01-00547), program of fundamental research of the Department for Informational Technologies and Computation Systems of the Russian Academy of Sciences (contract No 3.2/03), Russian Science Support Foundation and partly funded by the EU as part of the POSITIF project (contract No. IST-2002002314) and RE-TRUST (contract No. 021186-2). Author thanks Alexander Ulanov for software development and testing.

\section{REFERENCES}

[1] T. Back, D.B. Fogel, Z. Michalewicz. Evolutionary computation. Vol. 1. Basic algorithms and operators. Institute of Physics Publishing, 2000.

[2] E. Charniak, R.P. Goldman. A Bayesian Model of Plan recognition. Artificial Intelligence, vol. 64, no. 1, 1993.

[3] S. Chen, Q. Song. Perimeter-Based Defense against High Bandwidth DDoS Attacks. IEEE Transactions on Parallel and Distributed Systems, vol. 16, no. 7, 2005.

[4] P. Cohen, H.J. Levesque. Teamwork. Nous, no. 35, 1991.

[5] V.V. Druzhinin, D.S. Kontorov, M.D. Kontorov. Introduction into conflict theory. Moscow, Radio i svyas', 1989 (in Russian).

[6] T.Gamer, M.Scholler, R.Bless. A Granularityadaptive System for in-Network Attack Detection. Proceedings of the IEEE / IST 
Workshop on Monitoring, Attack Detection and Mitigation. 2006.

[7] C.W. Geib, R.P. Goldman. Plan recognition in intrusion detection systems. DARPA Information Survivability Conference and Exposition, DARPA and the IEEE Computer Society, 2001.

[8] V. Gorodetski, I. Kotenko. Conceptual foundations of stochastic simulation in the Internet. Proceedings of system analysis institute of RAS, vol.9, Moscow, URSS, 2005 (in Russian).

[9] B. Grosz, S. Kraus. Collaborative Plans for Complex Group Actions. Artificial Intelligence, vol. 86, 1996.

[10] D. Gu, E. Yang. Multiagent Reinforcement Learning for Multi-Robot Systems: A Survey. Technical Report of the Department of Computer Science, University of Essex, CSM404, 2004.

[11] P. Horn. Autonomic Computing: IBM's Perspective on the State of Information Technology. http://www.research.ibm.com/ autonomic/manifesto/autonomic_computing. pdf.

[12] J. Ioannidis, S.M. Bellovin. Implementing Pushback: Router-Based Defense Against DDoS Attacks. Symposium of Network and Distributed Systems Security (NDSS), California. 2002.

[13] Y. Ishida. Immunity-Based Systems A Design Perspective. Springer Verlag, 2004.

[14] C. Jin, H. Wang, K.G. Shin. Hop-count filtering: An effective defense against spoofed DDoS traffic. Proceedings of ACM Conference on Computer and Communications Security. 2003.

[15] J.O. Kephart, D.M. Chess. The Vision of Autonomic Computing. IEEE Computer Magazine, no. 1, 2003.

[16] A. Keromytis, V. Misra, D. Rubenstein. SOS: Secure Overlay Services. ACM SIGCOMM'02, Pittsburgh, PA. 2002.

[17] I.V. Kotenko. Agent-Based Modeling and Simulation of Cyber-Warfare between Malefactors and Security Agents in Internet. 19th European Simulation Multiconference "Simulation in wider Europe". 2005.

[18] I.V. Kotenko, A.V. Ulanov. Agent-based simulation of DDOS attacks and defense mechanisms. Journal of Computing, Vol.4, Issue 2, 2005.

[19] I. Kotenko, A. Ulanov. Agent Teams in Cyberspace: Security Guards in the Global Internet. CYBERWORLDS. 2006.

[20] I. Kotenko. Multi-agent Modelling and Simulation of Cyber-Attacks and Cyber-
Defense for Homeland Security // Proceedings of IDAACS'2007. Dortmund, Germany, 2007.

[21] V.A. Lefevre. Reflexion. Moscow, KognitoCenter, 2003 (in Russian).

[22] P. Mahadevan, D. Krioukov, M. Fomenkov, B. Huffaker, X. Dimitropoulos, K. Claffy, and A. Vahdat. Lessons from Three Views of the Internet Topology: Technical Report, CAIDA, 2005.

[23] J. Mirkovic, S. Dietrich, D. Dittrich, P. Reiher. Internet Denial of Service: Attack and Defense Mechanisms. Prentice Hall PTR, 2004.

[24] J. Mirkovic, M. Robinson, P. Reiher, G. Oikonomou. Distributed Defense Against DDOS Attacks. Technical Report CIS-TR2005-02. University of Delaware. 2005.

[25] M. Negoita, D. Neagu, V. Palade. Computational Intelligence Engineering of Hybrid Systems. Springer Verlag, 2005.

[26] OMNeT++. http://www.omnetpp.org/

[27] C. Papadopoulos, R. Lindell, I. Mehringer, A. Hussain, R. Govindan. Cossack: Coordinated suppression of simultaneous attacks. DISCEX III. 2003.

[28] T. Peng, L. Christopher, R. Kotagiri. Protection from Distributed Denial of Service Attack Using History-based IP Filtering. IEEE Conference on Communications. 2003.

[29] K.S. Perumalla, S. Sundaragopalan. HighFidelity Modeling of Computer Network Worm. 20th Annual Computer Security Applications Conference (ACSAC'04), December 06-10, 2004.

[30] F. Silva, M. Endler, F. Kon, R.H. Campbell, M.D. Mickunas. Modeling Dynamic Adaptation of Distributed Systems. Technical Report UIUCDCS-R-2000-2196, University of Illinois at Urbana-Champaign, 2000.

[31] M. Tambe. Towards flexible teamwork. Journal of AI Research, vol. 7, 1997.

[32] M. Vilain. Getting Serious about Parsing Plans: A Grammatical Analysis of Plan Recognition. Proceedings of the Eighth National Conference on Artificial Intelligence, Cambridge, MA, 1990.

[33] R. Want, T. Pering, D. Tennenhouse. Comparing autonomic and proactive computing. IBM Systems Journal, vol.42, no.1, 2003.

[34] M.P. Wellman, D.V. Pynadath. Plan Recognition under Uncertainty. 1997.

[35] C.C. Zou, N. Duffield, D. Towsley, W. Gong. Adaptive Defense against Various Network Attacks. IEEE Journal on Selected Areas in Communications: High-Speed Network Security (J-SAC), vol. 24, no. 10, 2006. 


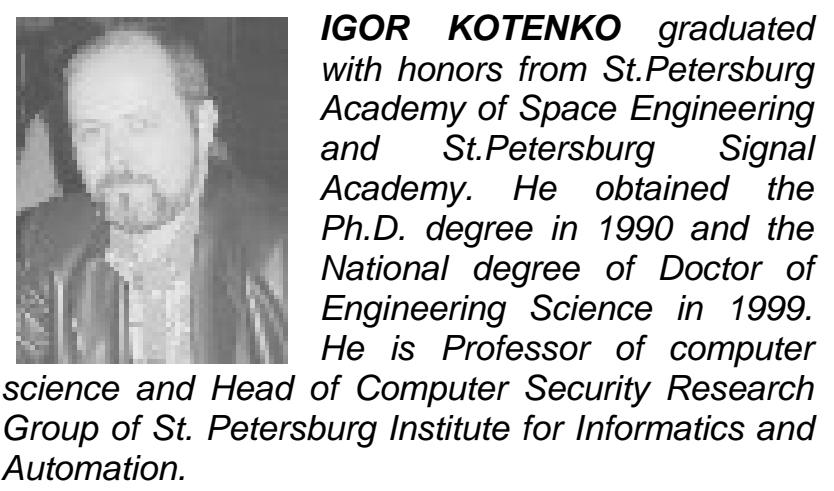

